\title{
Telemedicina durante la pandemia por COVID-19
}

\section{Telemedicine during the COVID-19 pandemic}

\author{
Sergio Monraz-Pérez,* Adrián Pacheco-López, Armando Castorena-Maldonado,* \\ Rosaura Esperanza Benítez-Pérez,* Ireri Thirión-Romero,* \\ Erika del Carmen López-Estrada,* Maribel Mateo-Alonso, \\ José Omar Barreto-Rodríguez, Ricardo Stanley Vega-Barrientos, ${ }^{*}$ José Luis Sandoval-Gutiérrez, \\ Sebastián Rodríguez-Llamazares, ${ }^{*}$ Justino Regalado-Pineda,* Jorge Salas-Hernández, \\ Patricio Santillán-Doherty, ${ }^{*}$ Miguel Ángel Salazar-Lezama, Juan Carlos Vázquez-García, José Rogelio Pérez-Padilla* \\ *Instituto Nacional de Enfermedades Respiratorias Ismael Cosío Villegas. Ciudad de México, México. \\ ${ }^{\ddagger}$ Centro Nacional de Excelencia Tecnológica en Salud, Ciudad de México, México.
}

\begin{abstract}
RESUMEN. La telemedicina a nivel mundial y en México ha sido de gran utilidad durante la pandemia por COVID-19. Poder prestar servicios de atención médica donde la distancia es un factor crítico, en un momento en el que se encuentran saturados los servicios de salud, y donde la atención presencial implica un riesgo, tanto para el proveedor de atención en salud como para los pacientes, ha sido indispensable durante la pandemia. La rapidez con la que se han implementado servicios de telemedicina a nivel mundial ha sido muy diferente. Aún quedan grandes retos por resolver para poder brindar este tipo de atención a nivel mundial. Cabe resaltar que la telemedicina viene a complementar la atención del paciente, más que a sustituir la habitual atención presencial.
\end{abstract}

Palabras clave: Telemedicina, telesalud, e-salud, COVID-19.

\section{INTRODUCCIÓN}

En diciembre de 2019, en Wuhan, provincia de Hubei en China, ocurrió una serie de casos graves de neumonía de etiología desconocida, lo cual alertó a la Organización Mundial de la Salud (OMS) el 31 de diciembre del 2019. Posteriormente, se identificaría al coronavirus tipo 2 del síndrome respiratorio agudo grave (SARS-CoV-2), con un origen zoonótico como causante de la enfermedad después

Correspondencia:

Dr. Sergio Monraz-Pérez

Instituto Nacional de Enfermedades Respiratorias

Ismael Cosío Villegas. Ciudad de México, México.

Correo electrónico: doctor.monraz@gmail.com

Recibido: 15-II-2021; aceptado: 24-III-2021.

Citar como: Monraz-Pérez S, Pacheco-López A, Castorena-Maldonado A, Benítez-Pérez RE, Thirión-Romero I, López-Estrada EC, et al. Telemedicina durante la pandemia por COVID-19. Neumol Cir Torax. 2021; 80 (2): 132-140. https://dx.doi.org/10.35366/100996
ABSTRACT. Telemedicine worldwide and in Mexico has been very useful during the COVID-19 pandemic. Being able to provide health care services where distance is a critical factor, at a time when health services are saturated, and where face-to-face care implies a risk for both the health care provider and the patients, it has been indispensable during the pandemic. The speed with which telemedicine services have been implemented globally has been very different. There are still great challenges to be solved in order to provide this type of care worldwide. It should be noted that telemedicine complements patient care, rather than replacing the usual face-to-face care.

Keywords: Telemedicine, telehealth, e-health, COVID-19.

descrita como COVID-19 (coronavirus disease 2019). La transmisión de este virus ocurre de persona a persona a través de aerosoles y gotas de personas con la enfermedad, así como a través del contacto con fómites. ${ }^{1}$

Dicha enfermedad se extendió rápidamente a nivel mundial, para el día 10/diciembre/2020 había 68'165,877 casos confirmados y 1'557,385 muertes. ${ }^{2}$ En México, para el día 9/diciembre/2020 se tenían 1'205,229 casos totales; 391,101 sospechosos totales y 111,655 defunciones. ${ }^{3}$

Esta pandemia ha ocasionado una crisis en los sectores de salud, social, económico y político. Durante las pandemias, la demanda de atención en los hospitales se incrementa de manera exponencial, y los recursos humanos con los que se cuentan para brindar dicha atención disminuyen, debido a enfermedad o por disposiciones gubernamentales. Hasta los insumos más básicos escasean en la población (medicamentos, equipos de protección personal, ventiladores, etc.) y se incrementa aún más la desigualdad para las personas que viven en comunidades rurales y de bajos recursos. ${ }^{4}$ 
En estados de emergencia y situaciones donde se declara una pandemia, los sistemas de salud pueden verse rebasados frente a la demanda de atención de la población. Las teleconsultas son una forma segura y efectiva para la evaluación de casos sospechosos, guían el diagnóstico y tratamiento del paciente, minimizando el riesgo de los trabajadores de salud de contagiarse. La teleconsulta, a veces denominada consulta remota, se refiere a las interacciones que ocurren entre un médico y un paciente con el fin de proporcionar asesoramiento diagnóstico o terapéutico a través de medios electrónicos. Algunos de los requisitos mínimos para poder otorgar teleconsulta es contar con conocimientos básicos en el uso de tecnologías, comprender las limitaciones inherentes a la teleconsulta y saber cuándo es preferible la atención presencial (cara a cara). ${ }^{5}$ A continuación, presentamos algunas implementaciones de la telemedicina a nivel mundial y en México durante la pandemia, sus beneficios y retos.

\section{TELEMEDICINA A NIVEL MUNDIAL DURANTE LA PANDEMIA POR COVID-19}

La OMS define a la telemedicina como «Aportar servicios de salud, donde la distancia es un factor crítico, por cualquier profesional de la salud, usando las nuevas tecnologías de la comunicación para el intercambio válido de información en el diagnóstico, el tratamiento y la prevención de enfermedades o lesiones, investigación y evaluación, y educación continuada de los proveedores de salud, todo con el interés de mejorar la salud de los individuos y sus comunidades». ${ }^{6}$

Establecer un programa de telemedicina, al igual que los programas de salud digital requiere de un apropiado ecosistema e incluye varias fases para su desarrollo, un plan estratégico, infraestructura, capacitación al personal, administración del cambio, mantenimiento y evaluación; ${ }^{7}$ por lo tanto, los países que ya contaban con servicios de telemedicina se encontraron en una posición más favorable para la atención de pacientes con COVID-19 por este medio.

En el Oeste de China, días después de que se notificaran los primeros casos, un grupo experto en respuesta rápida a desastres inició con un programa de teleeducación para trabajadores de la salud. Para el 26 de enero de 2020 generaron una red de servicio 5G para proveer de servicios de telemedicina a la población, así como para el trabajo multidisciplinario entre los hospitales, y también para la prescripción de medicamentos y entrega de los mismos. ${ }^{8}$

En Estados Unidos (EU), el Sistema de Cuidados a la Salud de los Veteranos (Veterans Affairs Healthcare System) se está enfrentando a múltiples retos de atención en salud derivados de la pandemia por COVID-19, como son continuar brindando atención a pacientes con enfermedades mentales y otras patologías no COVID-19, prevenir el conta- gio dentro de sus instalaciones de pacientes y personal de la salud, y proveer de atención médica a aquellos ciudadanos que se han mantenido en casa siguiendo las órdenes de salud pública de su país, antes brindaba ya atención a través de telemedicina; sin embargo, tuvo que incrementar la atención remota para lograr enfrentar estos retos. El número semanal de atenciones a pacientes con enfermedades mentales se incrementó de 7,442 en marzo de 2020 a 52,609 para finales de abril de 2020. En las mismas fechas, los cuidados de atención primaria incrementaron la atención por videollamada semanal de 1,102 a 13,068 y para cuidados de rehabilitación y cuidados de especialistas de 2,533 a 5,833. El número de clínicos utilizando telemedicina y videovisitas incrementó de 10,542 a 12,880 en atención primaria, de 8,599 a 11,173 en cuidados a la salud mental, y de 2,533 a 5,833 en cuidados de especialistas. El uso de atención por vía telefónica incrementó en 131\%, pasando de un promedio semanal de 327,180 a $756,195 .{ }^{9}$ En EU existen más de 50 sistemas de salud con programas en telemedicina como son: Jefferson Health, Mount Sinai, Kaiser Permanente, Cleveland Clinic, Providence, entre otros. Algunas de las estrategias que han implementado durante esta pandemia son la priorización de la atención de pacientes antes de que lleguen a urgencias (forward triage), la inteligencia artificial (uso de asistentes cognitivos) para identificar pacientes con riesgos moderados-altos y canalizarlos para ser valorados en urgencias. También permitir a los pacientes agendar citas para toma de muestras diagnósticas para COVID-19, interrogatorio médico a distancia, monitorización a distancia de pacientes en unidades de cuidados intensivos, coordinar atención de pacientes en su casa, trabajo del personal de salud desde casa para aquellos que se encuentran en riesgo, acceso del paciente a su expediente clínico electrónico y el seguimiento de pacientes. ${ }^{10}$

En Francia, el Ministerio de Salud firmó un decreto el 9 de marzo de 2020, en el cual se autorizó el reembolso de las teleconsultas por video para pacientes con sospecha y aquellos confirmados con COVID-19 en todo el país. Más tarde se autorizó el reembolso para enfermería, parteras y terapistas de lenguaje. ${ }^{11}$

En Alemania se realizó una encuesta nacional en línea entre marzo y abril de 2020 a 2,827 médicos (65\%), enfermeras (30\%) y profesionales de la salud (5\%) a lo largo de 14 días. El 19.6\% de los encuestados ya utilizaban telemedicina y $40.2 \%$ referían un uso parcial. El $39 \%$ de los que respondieron consideraron que la importancia de la telemedicina es alta. Este dato está inversamente relacionado con la edad del entrevistado, posiblemente asociado a la experiencia en el uso de tecnologías de información. Debido a que la mayoría de los hospitales ya contaban con plataformas establecidas, es más común utilizar telemedicina en el manejo intrahospitalario que en el contexto ambulatorio. Asimismo, quienes laboran en el área privada experimentan 
Sistema de telemedicina en hospitales y unidades públicas

Tasa por cada 100,000 habitantes $\geq 15$ años de edad (INEGl: censo poblacional 2020)

Análisis geoespacial de los sistemas de telemedicina y plazas de diversas especialidades médicas

Modelo espacial autorregresivo

Estimación por mínimos cuadrados
Número de estados $=32$

Wald $\chi^{2}(5)=23.97$

Prob $>\chi^{2}=0.0002$

Pseudo R2 $=0.2726$

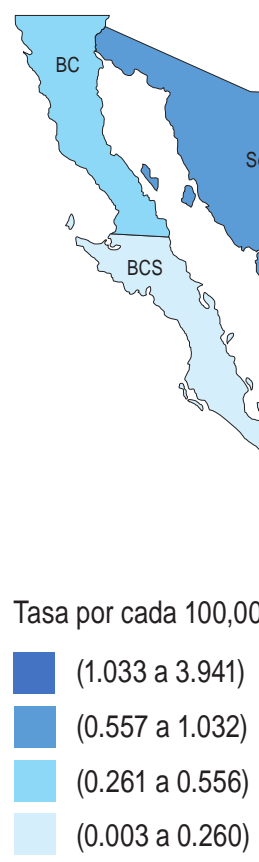

menos obstáculos regulatorios o técnicos, comparado con los hospitales gubernamentales. ${ }^{12}$

Otros ejemplos de uso de la telemedicina a nivel mundial durante la pandemia ha sido la implementación de aplicaciones para celulares, con la finalidad de localizar y alertar de ciudadanos sospechosos de haber contraído COVID-19. Esto se ha implementado de forma obligatoria en algunos países como China, Israel e Irán, y de forma voluntaria en naciones como Gran Bretaña. ${ }^{13}$

\section{TELEMEDICINA EN MÉXICO DURANTE LA PANDEMIA POR COVID-19}

En México se confirmó el primer caso de COVID-19 el día 27 de febrero de 2020 en el Instituto Nacional de Enfermedades Respiratorias Ismael Cosío Villegas (INER), Ciudad de México. Con el fin de hacer frente a la contingencia, un número considerable de entidades federativas a través de los Servicios Estatales de Salud implementaron proyectos de telemedicina para dar atención a la población de una manera segura. Para diciembre de 2019, de acuerdo con la información encontrada en el Observatorio de Telesalud, 23 entidades federativas contaban con infraestructura y programas consolidados de telesalud ${ }^{14}$ lo que facilitó incorporar acciones específicas de telemedicina para enfrentar la contingencia.

\begin{tabular}{|l|c|c|c|c|}
\hline Sistema de telemedicina & Coeficiente & $\mathbf{P ~ > ~ z ~}$ & \multicolumn{2}{|c|}{$\begin{array}{c}\text { [Intervalo de } \\
\text { confianza 95\%] }\end{array}$} \\
\hline Tasa telemedicina & & & & \\
Tasa otorrinolaringología & 0.131 & 0.260 & -0.097 & 0.360 \\
Tasa neumología & 0.449 & 0.222 & -0.272 & 1.17 \\
Tasa infectología & -0.075 & 0.883 & -1.084 & 0.933 \\
Tasa rehabilitación & -0.245 & 0.525 & -1.003 & 0.511 \\
Constante & -0.028 & 0.906 & -0.500 & 0.443 \\
\hline W (Moran) & & & & \\
Tasa de telemedicina & 0.789 & $\mathbf{0 . 0 1 2}$ & $\mathbf{0 . 1 7 4}$ & 1.404 \\
\hline Wald para análisis espacial: $\chi^{2}(1)=6.32$ & \multicolumn{4}{|l|}{ Prob $>\chi^{2}=0.0119$} \\
\hline
\end{tabular}

Figura 1:

Análisis geoespacial del número de sistemas de telemedicina en el sector público en México.

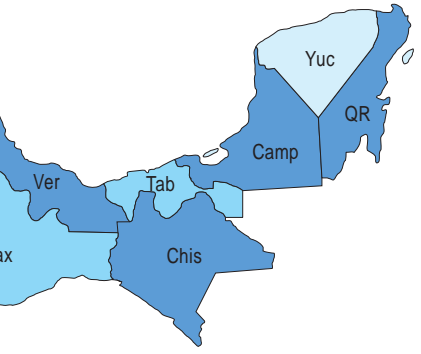

ANÁLISIS GEOESPACIAL

Con el propósito de conocer la distribución territorial de los sistemas o unidades que cuentan con telemedicina en el país y la relación que tiene con el número de diversas especialidades médicas asociadas con la atención de pacientes contagiados por COVID-19, se confeccionó un mapa bajo el marco de georreferencia estatal del Instituto Nacional de Estadística y Geografía (INEGI). El número de sistemas de telemedicina y especialidades médicas fueron obtenidos de la base de datos de recursos humanos de la Dirección General de Información en Salud de la Secretaría de Salud del año 2019 y, finalmente, se calculó la tasa por cada 100,000 habitantes, descargamos el censo poblacional del año 2020 contenido en el INEGI.

Una vez identificadas las variables clave de estado, sector, nombre de estado y tipo de establecimiento de salud, se comprobó que dichas variables fueran consistentes entre sí y consideradas como los identificadores oficiales. Para realizar el análisis geoespacial, se utilizó el método de modelos espaciales autorregresivos de mínimos cuadrados y la confección de los mapas se realizó por el método de coropletas. En la figura 1 se puede observar que la distribución estatal de los sistemas de telemedicina sigue un patrón estadísticamente diferente (M) con dirección hacia el este del país, debido a 
que los estados con mayores números son Chiapas, Veracruz, San Luis Potosí, Coahuila y Zacatecas, con tasas de $3.9,1.7,1.5,1.3,1.18$, respectivamente, por cada 100,000 habitantes $\geq 15$ años de edad, pero no encontramos una asociación territorial con las especialidades de neumología, otorrinolaringología, infectología ni rehabilitación.

La tabla 1 muestra las tasas de las especialidades médicas que podrían estar más relacionadas con los cuidados de pacientes con COVID-19 en el sistema público nacio- nal, no encontramos una diferencia estadística, pero es notorio que en la Ciudad de México existen las tasas más elevadas de especialistas, excepto por la tasa de sistemas de telemedicina.

Las medidas asumidas por las autoridades sanitarias para contener la velocidad del contagio incluyeron la suspensión de consultas presenciales y cirugías efectivas por el alto riesgo de contagio, tanto para el paciente y sus familiares como para el personal de salud; por lo que, con el fin de

Tabla 1: Sistema de telemedicina y su relación con diversas especialidades médicas de atención post-COVID.

Sistema de telemedicina y plazas de especialidades médicas relacionadas con la atención en la pandemia por COVID-19. Tasa por cada 100,000 habitantes $\geq 15$ años de edad (INEGl: censo poblacional 2020).

\begin{tabular}{|c|c|c|c|c|c|c|c|c|c|}
\hline & $\begin{array}{l}\text { Sistema de } \\
\text { telemedi- } \\
\text { cina }\end{array}$ & $\begin{array}{l}\text { Neumo- } \\
\text { logía }\end{array}$ & $\begin{array}{l}\text { Otorrinola- } \\
\text { ringología }\end{array}$ & $\begin{array}{l}\text { Infec- } \\
\text { tología }\end{array}$ & $\begin{array}{l}\text { Rehabili- } \\
\text { tación }\end{array}$ & $\begin{array}{l}\text { Cardio- } \\
\text { logía }\end{array}$ & $\begin{array}{l}\text { Neuro- } \\
\text { logía }\end{array}$ & $\begin{array}{l}\text { Psi- } \\
\text { quiatría }\end{array}$ & $\begin{array}{l}\text { Medicina } \\
\text { Interna }\end{array}$ \\
\hline Aguascalientes & 0.003 & 0.008 & 0.025 & 0.005 & 0.012 & 0.030 & 0.010 & 0.021 & 0.114 \\
\hline Baja California Norte & 0.481 & 1.057 & 3.460 & 0.577 & 2.018 & 3.845 & 1.826 & 2.115 & 18.935 \\
\hline Baja California Sur & 0.208 & 0.104 & 0.519 & 0.035 & 0.173 & 0.415 & 0.069 & 0.311 & 2.525 \\
\hline Campeche & 0.998 & 0.665 & 1.663 & 0.166 & 0.665 & 0.831 & 0.333 & 2.494 & 13.302 \\
\hline Chiapas & 3.941 & 1.971 & 3.941 & 1.433 & 2.508 & 5.732 & 3.762 & 2.150 & 27.946 \\
\hline Chihuahua & 1.086 & 0.318 & 1.192 & 0.212 & 0.662 & 1.113 & 0.742 & 0.768 & 5.035 \\
\hline Ciudad de México & 1.036 & 7.608 & 9.180 & 4.786 & 9.252 & 15.610 & 7.430 & 16.860 & 37.863 \\
\hline Coahuila de Zaragoza & 1.310 & 2.183 & 8.294 & 0.582 & 2.765 & 9.022 & 3.783 & 6.257 & 32.157 \\
\hline Colima & 0.086 & 0.172 & 0.517 & 0.043 & 0.474 & 0.431 & 0.215 & 0.388 & 3.229 \\
\hline Durango & 0.278 & 0.093 & 0.212 & 0.066 & 0.132 & 0.291 & 0.159 & 0.344 & 1.720 \\
\hline Guanajuato & 0.758 & 0.910 & 3.640 & 0.682 & 1.592 & 5.005 & 1.517 & 4.171 & 22.825 \\
\hline Guerrero & 0.618 & 0.154 & 0.463 & 0.000 & 0.441 & 0.309 & 0.199 & 0.265 & 3.222 \\
\hline Hidalgo & 0.279 & 0.160 & 0.718 & 0.040 & 0.439 & 0.599 & 0.319 & 0.718 & 5.428 \\
\hline Jalisco & 0.524 & 0.873 & 4.844 & 1.484 & 1.702 & 4.451 & 1.440 & 4.320 & 15.885 \\
\hline Michoacán de Ocampo & 0.109 & 0.093 & 0.319 & 0.062 & 0.132 & 0.264 & 0.155 & 0.218 & 1.468 \\
\hline Morelos & 0.145 & 0.145 & 0.609 & 0.203 & 0.290 & 0.580 & 0.377 & 0.348 & 3.883 \\
\hline Estado de México & 0.577 & 0.512 & 1.618 & 0.320 & 0.881 & 1.585 & 0.881 & 1.634 & 12.348 \\
\hline Nayarit & 0.532 & 0.266 & 1.198 & 0.333 & 0.665 & 0.599 & 0.266 & 0.532 & 3.792 \\
\hline Nuevo León & 3.316 & 4.422 & 7.296 & 1.990 & 5.196 & 7.628 & 5.527 & 9.839 & 27.969 \\
\hline Oaxaca & 0.432 & 0.159 & 0.545 & 0.182 & 0.273 & 0.522 & 0.386 & 0.500 & 3.657 \\
\hline Puebla & 0.536 & 0.502 & 1.541 & 0.268 & 1.072 & 1.206 & 0.737 & 1.474 & 7.269 \\
\hline Querétaro & 0.229 & 0.167 & 0.396 & 0.042 & 0.229 & 0.354 & 0.271 & 0.541 & 2.436 \\
\hline Quintana Roo & 0.784 & 0.112 & 0.896 & 0.056 & 0.504 & 0.560 & 0.168 & 0.560 & 5.378 \\
\hline San Luis Potosí & 1.571 & 0.428 & 1.428 & 0.286 & 1.214 & 1.714 & 1.214 & 2.713 & 8.925 \\
\hline Sinaloa & 0.620 & 0.716 & 2.290 & 0.477 & 1.145 & 1.622 & 1.050 & 1.479 & 9.209 \\
\hline Sonora & 0.746 & 0.702 & 2.018 & 0.570 & 0.921 & 2.368 & 0.921 & 2.105 & 11.184 \\
\hline Tabasco & 0.495 & 0.225 & 1.036 & 0.315 & 0.810 & 1.216 & 0.630 & 2.161 & 6.664 \\
\hline Tamaulipas & 1.029 & 0.915 & 2.630 & 0.286 & 1.086 & 2.859 & 1.258 & 1.830 & 12.979 \\
\hline Tlaxcala & 0.113 & 0.038 & 0.264 & 0.113 & 0.151 & 0.339 & 0.151 & 0.264 & 2.409 \\
\hline $\begin{array}{l}\text { Veracruz de Ignacio de } \\
\text { la Llave }\end{array}$ & 1.708 & 1.708 & 6.432 & 1.306 & 4.221 & 5.829 & 3.316 & 5.226 & 34.671 \\
\hline Yucatán & 0.246 & 0.246 & 0.459 & 0.115 & 0.443 & 0.705 & 0.410 & 0.590 & 3.327 \\
\hline Zacatecas & 1.187 & 0.565 & 0.565 & 0.170 & 0.170 & 1.017 & 0.226 & 0.339 & 4.408 \\
\hline Mediana & 0.556 & 0.373 & 1.195 & 0.240 & 0.665 & 0.065 & 0.520 & 1.121 & 6.046 \\
\hline
\end{tabular}


mantener la continuidad de la atención, las consultas por telemedicina cobraron mayor importancia.

Las instituciones de salud federales y estatales realizaron acciones de atención médica y de apoyo a la salud basadas en modelos tecnológicos. Además de las consultas a distancia se incluyeron acciones de prevención y asesoría de temas relacionados a COVID-19 y sus consecuencias, así como para el seguimiento de enfermedades crónicas, control de embarazo y salud mental. La colaboración entre los profesionales de la salud mediante el uso de estas tecnologías se incrementó considerablemente, la capacitación a distancia, mentoría e interconsulta fueron algunas de las actividades que destacan en este período.

De acuerdo con información compartida por los coordinadores estatales de telesalud, ${ }^{14}$ en Sinaloa del 27 de marzo al 21 de junio de 2020 se atendieron 31,809 pacientes, en promedio 365 diarios, operando 24 horas al día. Para septiembre de 2020 se habían contabilizado más de 62,805 casos georreferenciados y en seguimiento, con inteligencia aplicada por colonia para diseñar estrategias de prevención y atención efectivas. La Secretaría de Salud de Hidalgo a través de los sistemas de información radiológica ha interpretado a distancia más de 6 mil estudios radiológicos para descartar neumonía y más de 1,200 videollamadas de seguimiento para COVID-19, y con aplicación con asistentes cognitivos se atendieron cerca de 470 mil interacciones con ciudadanos para revisar temas relacionados. Dentro de las estrategias que ha implementado el Departamento de Telesalud de la Secretaría de Salud de Nuevo León resaltan: tótems de videoconferencia, pase de visita virtual, visita familiar virtual, plataforma de teleeducación, plataforma de recopilación de datos hospitalarios y unificación de la información clínica electrónica. De manera general, se reportan durante el 2020 más de 5.5 millones de acciones de telemedicina contra COVID-19 de las Secretarías de Salud de 20 entidades federativas, entre las que destacan teleconsulta, asesorías médicas, interconsulta a distancia, diagnóstico a distancia y seguimiento remoto de pacientes.

El uso de la teleasistencia en salud mental en México es positivo, hace eficiente el tiempo del paciente y del personal de salud. ${ }^{14}$ Según un estudio del Centro de Investigación Económica y Presupuestaria, una forma de incrementar el acceso de los servicios para salud mental es a través de la telemedicina. Antes de la pandemia de 2013 a 2019, las consultas de psiquiatría vía telemedicina en los servicios públicos disminuyeron 87.3\%, pasaron de 70 mil 396 en 2013 a 8 mil 932 en 2019.15

Instituciones federales como el Instituto Nacional de Cardiología Ignacio Chávez atiende a más de 120 mil consultas al año, en esta época de contingencia por COVID-19 se atendieron a distancia en las 14 clínicas de atención cardiovascular aplicando encuestas para la detección de casos sospechosos. Además, se establecieron, apoyados en tecnología de información y comunicaciones, grupos de trabajo para optimizar la estancia en hospital y casa. ${ }^{16}$

Las soluciones digitales basadas en web han sido empleadas para difundir información entre los ciudadanos, además del uso de redes sociales para subir videos informativos, infografías, uso de asistentes cognitivos para responder preguntas comunes, mapas de georreferenciación, ubicación de lugares para la aplicación de pruebas y camas disponibles han sido utilizados por diversas instituciones de salud. La Secretaría de Salud de la Ciudad de México utiliza portales en web, plataformas de mensajería instantánea, llamadas telefónicas y cuestionarios digitales para estar en contacto con la población. ${ }^{17}$ Otras entidades federativas han implementado soluciones similares al Gobierno de la Ciudad de México, entre ellas Baja California Sur, Durango, Chihuahua, Veracruz, Yucatán y Zacatecas por citar algunos.

\section{Videoconferencias y telemedicina}

Una práctica muy común y cada vez más inherente de las actividades de los profesionales de la salud, es el uso de las plataformas colaborativas y de videoconferencia para la capacitación del personal de salud. Tanto en tiempo real como de forma diferida, las sesiones virtuales para el desarrollo de talleres, clases y adiestramiento fueron una de las herramientas más utilizadas durante la pandemia de COVID-19. Si bien la calidad de las redes de Internet es todavía limitada en muchos de las unidades médicas ubicadas en las zonas rurales del país, en aquellos lugares con buen acceso y cobertura de Internet, las plataformas virtuales fueron altamente utilizadas para capacitarse en el manejo del paciente COVID-19.

En el INER, hubo una rápida respuesta en la implementación de un servicio de telemedicina, con apoyo de una plataforma donada por Cisco-WebEx a través de la cual se programan televisitas (videollamada del familiar con el paciente), informe médico (médico-familiar del paciente) y atención domiciliaria (orientación médica para pacientes no hospitalizados) (Figura 2). Asimismo, al ser reconvertidos como Hospital COVID-19, nuestra atención se enfocó inicialmente en pacientes críticos con dicha patología, y se decidió utilizar esta plataforma para dar atención también a pacientes con otras enfermedades (Figura 3).

La implementación de la telemedicina en el instituto se ha enfrentado a algunos retos, como son la falta de conocimiento en el uso de las tecnologías de la información y la comunicación (TIC), opacidad en los temas legales que conlleva la utilización, consideraciones éticas y bioéticas, técnicas, conectividad con el usuario y de implementación de un nuevo proceso. A pesar de dichas dificultades, el INER ha logrado incrementar semanalmente el número de videollamadas, manteniendo la satisfacción del usuario en la calidad de la atención médica (Figura 3). 
Neumol Cir Torax. 2021; 80 (2): 132-140

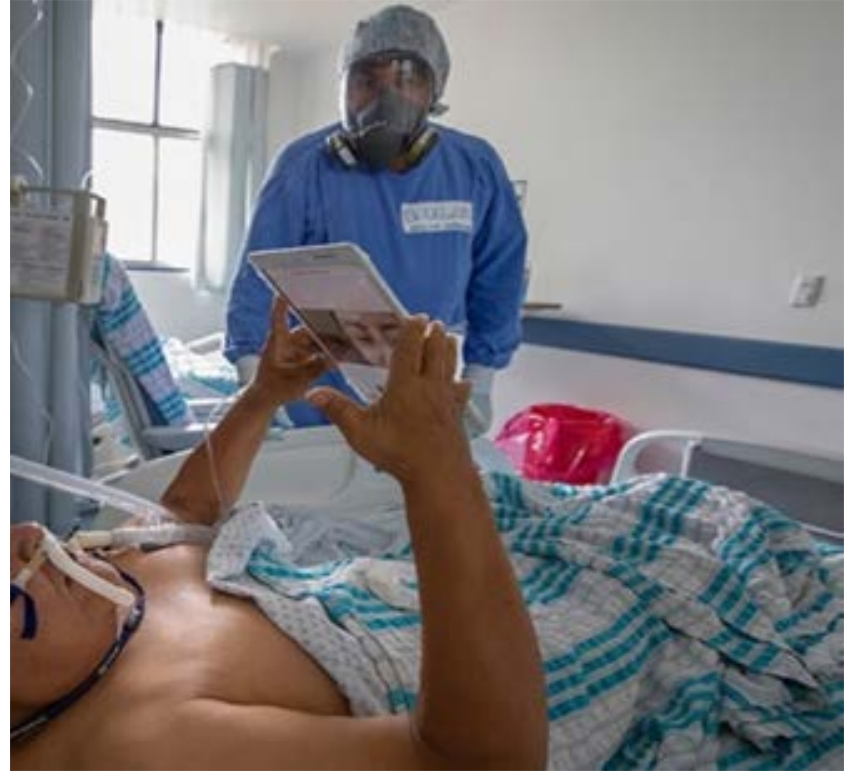

Figura 2: Televisita. Mediante el uso de la plataforma Cisco y dispositivos electrónicos se acerca al familiar con el paciente.

\section{BENEFICIOS DE LA TELEMEDICINA}

Uno de los factores clave para frenar la transmisión del virus es el distanciamiento social. Para aquellas personas con síntomas y con la sospecha de tener COVID-19, la telemedicina ha ayudado en realizar la evaluación inicial y en orientar al paciente con base en el interrogatorio realizado. De igual forma, la telemedicina permite brindar atención a aquéllos que por ser personal vulnerable o de alto riesgo, podría ser un riesgo su atención presencial. La telemedicina se ha utilizado efectivamente en otras situaciones de emergencia, como desastres naturales (temblores, huracanes), militares y otras pandemias. No obstante, a pesar de los beneficios, la implementación en algunos países ha sido lenta y fragmentada. ${ }^{18}$

Otros de los beneficios descritos sobre la telemedicina es la mejoría en el acceso a la información y educación continua, comunicación entre profesionales de la salud, información para pacientes y población en general, proveer de atención en lugares remotos o donde antes no se brindaba (accesibilidad), ahorro de tiempo y recursos para los pacientes, mejoría en la calidad de la atención, monitorización y diagnóstico de enfermedades, referencias inter e intrahospitalarias, interpretación de estudios diagnósticos, disminución en los costos de atención, entre otras. ${ }^{19}$

En México como en otros países, existe inequidad en el acceso a servicios de la salud, así como en la atención especializada. La implementación de tecnologías de la información en el campo de la salud ha facilitado proveer de atención y seguimiento a pacientes con enfermedades crónicas, así como educación y prevención de enfermedades, principalmente a estos pacientes que no cuentan con acceso a servicios de la salud. De esta forma, la telemedicina favorece la atención equitativa, sin importar la localización geográfica, reduce tiempos de espera y de traslados, previene desarrollo de enfermedades y complicaciones de las mismas, minimiza el número de referencias a hospitales de alta especialidad y, por último, facilita el acceso y continuidad de la atención de los pacientes (Tabla 2). ${ }^{20}$

\section{RETOS Y BARRERAS DE LA TELEMEDICINA}

De acuerdo con la OMS y la Organización Panamericana de la Salud (OPS) podemos clasificar a las barreras para la implementación de la telemedicina en cuatro grandes grupos: tecnología, organización, factor humano y económico. ${ }^{20}$

En cuanto a las tecnologías, uno de los principales problemas es la falta de infraestructura, equipos y capacitación del personal en su uso. Se suman el limitado acceso a Internet, personas que no cuentan con teléfonos celulares inteligentes o una computadora para llevar a cabo una videollamada, lo que conlleva que en ocasiones dicha videollamada termine en una llamada telefónica que aporta menos datos en la atención del paciente. Otro gran reto es asegurar la protección de los datos personales del paciente, así como la interoperabilidad, es decir, el acceso a esta información entre los diferentes hospitales del sistema de salud. Es por ello que se requiere que un mayor número de zonas geográficas cuente con Internet y la tecnología adecuada, así como educar al paciente y ayudarle a familiarizarse con el uso de tecnologías de la comunicación y perder el miedo a las mismas, también a asegurar la protección de los datos del paciente.

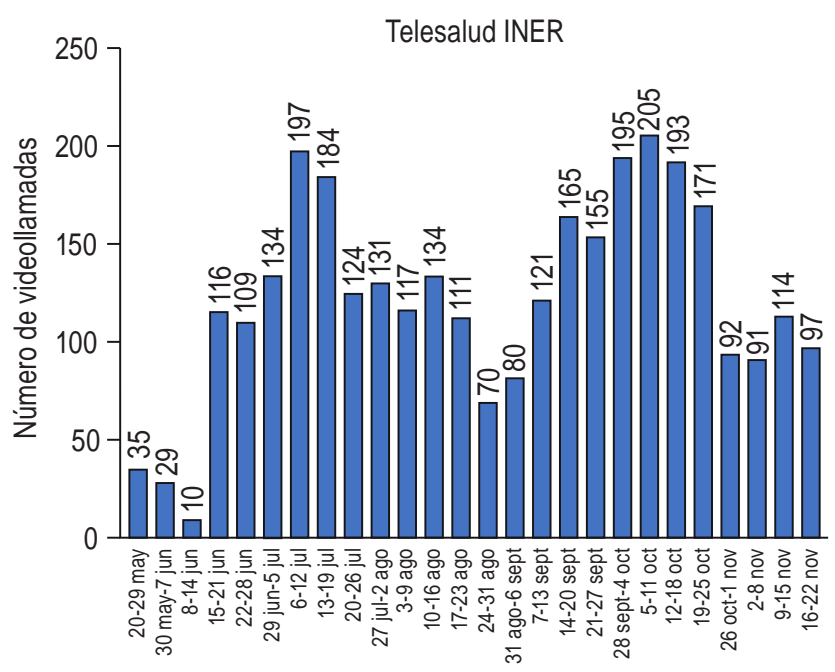

Figura 3: Número de videollamadas desde su implementación el día 20 de mayo de 2020, hasta la semana del 16 al 22 de noviembre de 2020. 
Tabla 2: Ventajas y desventajas de la telemedicina.

\begin{tabular}{|l|l|}
\hline Ventajas & Desventajas \\
\hline Mayor acceso a la atención & Supone una pérdida de la relación personal directa con el paciente \\
\hline Continuidad de la atención & $\begin{array}{l}\text { Poca interoperabilidad de los sistemas hospitalarios para compartir la } \\
\text { información del paciente }\end{array}$ \\
\hline Mayor diagnóstico y monitorización de enfermedades & Determinar el reembolso para la atención de pacientes \\
\hline Previene desarrollo de enfermedades y complicaciones de las mismas & Poblaciones sin acceso a Internet, dispositivos móviles o computadoras \\
\hline Reducción de hospitalizaciones y visitas a urgencias & $\begin{array}{l}\text { Falta de personal capacitado con un enfoque en el uso de tecnologías } \\
\text { de la información }\end{array}$ \\
\hline Disminución de los costos de atención & Infraestructura insuficiente en la mayoría de hospitales \\
\hline $\begin{array}{l}\text { Respuesta de las instituciones de salud a un nuevo tipo de paciente } \\
\text { que demanda acciones digitales para su atención }\end{array}$ & $\begin{array}{l}\text { Implicaciones éticas y legales aún con lagunas sobre el alcance de la } \\
\text { telemedicina }\end{array}$ \\
\hline $\begin{array}{l}\text { Mejora los procesos al incorporarse a un mundo digital con mayores } \\
\text { posibilidades de gestión de la información }\end{array}$ & \\
\hline $\begin{array}{l}\text { Empoderamiento de los pacientes al recibir atención médica mediante } \\
\text { dispositivos propios, información en salud en sus manos }\end{array}$ & \\
\hline
\end{tabular}

Otro gran desafío es la reorganización y rediseño del sistema de salud, ya que muchos países continúan funcionando completamente mediante la atención presencial. Se deben formar nuevos especialistas con un enfoque en el uso de las tecnologías de la información, capacitar a personal de salud y estudiantes de las diferentes áreas de salud, fomentar y apoyar trabajos de investigación. Asimismo, debe haber regulación y legislación en materia de telemedicina para facilitar su uso por los profesionales de la salud.

En cuanto al factor humano, el principal reto a vencer es la resistencia al cambio, lo cual suele ser multifactorial (p. ej. falta de capacitación en uso de tecnologías de la información, miedo a lo desconocido, cuestiones legales). Asimismo, tiene que ver con la reorganización de los sistemas de salud, ya que la mayoría del personal de la salud está acostumbrado a brindar atención de forma presencial, mientras que no hay mucha experiencia en la atención a distancia, que se explica por la falta de educación y entrenamiento en la mayoría de las universidades. Actualmente, también tiene que ver con la carga de trabajo a la cual se enfrentan los trabajadores de la salud y a sus intereses y creencias particulares (aquéllos que apoyan el uso de la telemedicina y los que se encuentran escépticos). Además, se requiere entrenamiento para estas nuevas tareas.

Por último, el factor económico para la implementación de servicios de telemedicina, así como para el reembolso de la atención y la sustentabilidad de los proyectos es uno de los más grandes retos. En algunos países se han ido venciendo estas barreras, p. ej. en EU los programas de cobertura social Medicare, Medicaid y otros proveedores locales, modificaron su política de reembolso durante la pandemia por COVID-19 a través del Acta de Asignacio- nes Suplementarias de Preparación y Respuesta Frente al Coronavirus (Coronavirus Preparedness and Response Supplemental Appropriations Act), así como relajar las restricciones actuales en el uso de telemedicina por el Ley de Portabilidad y Responsabilidad del Seguro de Salud (Health Insurance Portability and Accountability Act, HIPAA) cuya función, entre otras, es salvaguardar y proteger los datos del paciente. ${ }^{13}$

Para la adecuada implementación se requiere una estrategia que involucre a todo el sistema de salud, así como políticas públicas, adecuada legislación y apoyar a las instituciones de salud con la infraestructura adecuada (conectividad) y equipos de comunicación. Todo esto involucra tiempo al igual que recursos económicos.

Existen otras limitaciones como son la atención de enfermedades o condiciones del paciente en las que la atención no puede realizarse de forma remota, así como situaciones en las cuales se requieren estudios diagnósticos, que en ocasiones no pueden ser realizados de forma remota.

Algunas de las barreras más importantes para la difusión de la telemedicina, es la falta de estándares internacionales que guíen el uso de la misma en los diferentes países, así como la falta de disposiciones legales aplicables a los servicios remotos y la práctica de servicios de salud a distancia. En 2008, la Comisión Europea estableció el proyecto Telescope, que es una plataforma cuya intención es reunir las mejores prácticas y experiencias de la atención remota en Europa. Al finalizar este trabajo se creó el Código de Telesalud (Telehealth Code), instrumento concebido para proveer parámetros técnicos recomendables para asegurar y proveer atención de calidad a distancia. Se ha ido actualizando este código por grupos de Calidad en Telesalud. ${ }^{21}$ 
De igual forma se requiere de un compromiso por parte del paciente, contar con una actitud proactiva para el manejo de su enfermedad, además de familiarizarse con los recursos tecnológicos para poder ser atendido bajo esta modalidad.

El uso de las tecnologías de la información por sí mismas no deben catalogarse dentro de consideraciones éticas; más bien es el uso y la intención al cual se les dé y que debe tener siempre un enfoque centrado en el paciente. En cuanto a los puntos éticos más controvertidos para la implementación de la telemedicina se encuentran respetar la privacidad del paciente, la confidencialidad y la responsabilidad de proteger sus datos, así como respetar la autonomía del paciente a hacer uso o no de este tipo de atención remota. ${ }^{22}$ Se debe salvaguardar los datos personales y confidencialidad del paciente, así como obtener su consentimiento para la práctica de la telemedicina. También debemos tomar en cuenta la equidad en el acceso a la salud, y que las personas que habitan en regiones rurales cuentan con limitado acceso a servicios de salud y atención médica especializada que puede mejorarse con el uso de la telemedicina. Los principios éticos y legales que rigen a la atención de la práctica médica en telemedicina son igualmente válidos para la atención a distancia. ${ }^{23}$

En 1997, la Asociación Médica Finlandesa creó una guía ética para el uso de la telemedicina, donde se mencionaba que el uso de ésta debía ser restringida a situaciones en las cuales el paciente no puede ver personalmente a un proveedor de salud debido a limitaciones físicas, geográficas o de tiempo. ${ }^{24}$ La Asociación Médica Mundial (World Medical Association) publicó en su página de Internet, declaraciones de ética en la telemedicina, adoptados de su asamblea general en 2007 y modificados en 2018. ${ }^{25}$

Otro de los retos de la telemedicina supone una pérdida de la relación personal directa con el paciente, así como las implicaciones éticas, como son adaptarse a las regulaciones y permisos locales del uso de la telemedicina, asegurarse de mantener la confidencialidad del paciente, explicar al paciente cómo funciona la telemedicina, el derecho de los profesionales de la salud a ejercer su autonomía y decidir el método de atención de su preferencia (presencial, remoto), así como mantener un registro de las recomendaciones brindadas al paciente, asegurarse de que éste haya comprendido las instrucciones, identificar cuándo debe ser valorado de forma presencial y ejercer la práctica de la telemedicina en países donde esté facultado para ejercer la medicina. ${ }^{19,25}$

\section{DISCUSIÓN}

Implementar un servicio de telemedicina es un reto enorme, pues requiere de la participación de: 1) recursos humanos: personal médico, enfermería, técnicos en informática y sistemas, administrativos para la planeación, coordinación de agenda y cobros, centros de llamado y resolución de dudas; 2) infraestructura: acceso a Internet, computadoras o dispositivos inteligentes, telefonía, sistemas de geolocalización, protección de datos, alarmas, entre otros; y 3) actualización e innovación permanente que nos permitan optimizar tiempos y recursos, a través de la generación de algoritmos de inteligencia artificial y la generación de documentos o formatos electrónicos, entre ellos recetas e instructivos para los pacientes. Todo ello a expensas de una legislación adecuada apegada a las normas y derechos humanos universales de todo individuo.

No obstante, y pese al gran reto que se tiene, la implementación de telemedicina no sólo permite atención médica oportuna para poblaciones marginadas, sino que además mejora y desahoga servicios de salud a gran escala, impactando sustancialmente en la reducción de costos a la salud, tanto para las instituciones públicas como privadas de cualquier país. En un inicio la productividad puede ser baja en lo que el equipo humano se adapta al nuevo proceso; sin embargo, una vez instalado el servicio y tras la capacitación de los trabajadores de la salud como de los pacientes ya familiarizados con el uso de la telemedicina, se empieza a solicitar cada vez más y más este servicio. Sus ventajas son bastas y las limitantes resolutivas, por lo que el mayor impedimento está en nosotros mismos, en el miedo al cambio y a la visión futurista limitada. El cambio se puede lograr y nos lo ha dejado claro esta pandemia.

Particularmente en el INER, después de implementar el Servicio de Telemedicina, se identificaron otros servicios que se lograron ofrecer a través de esta plataforma y brindamos atención a zonas remotas que inicialmente no atendíamos. Ha sido apropiado ofrecer servicios de seguimiento a pacientes con enfermedades respiratorias crónicas que en momentos de la pandemia se encuentran abandonados y sin atención, además de que se ha optimizado el seguimiento de los pacientes egresados tras su curación de COVID-19.

Aún quedan algunas dificultades por resolver cómo el acceso libre a Internet y servicios de telecomunicación en las diferentes zonas geográficas, así como las implicaciones éticas y legales para poder otorgar de manera universal servicios de telesalud en los diferentes países.

La pandemia por COVID-19 ha puesto en manifiesto un nicho de oportunidad para las diferentes organizaciones en salud a nivel mundial, siendo un foco de alerta de que no estamos preparados para enfrentar situaciones como la actual y, por lo tanto, debemos gestionar la implementación y universalidad de la telesalud.

\section{CONCLUSIÓN}

La atención a distancia es una necesidad, no sólo por la pandemia de COVID-19, sino por la presencia de pacientes 
con limitado acceso a los servicios de salud, principalmente en zonas rurales alejadas y de difícil comunicación. Es indispensable que los servicios de salud desarrollen sistemas de telemedicina, y se vaya adecuando y perfeccionando a las necesidades locales. Hay que tener siempre en mente que los servicios de telemedicina vienen a complementar la atención estándar, más que a sustituirla, aún queda mucho esfuerzo por superar, como la inseguridad jurídica, respetar los principios éticos y unificar las mejores prácticas en telemedicina para la atención de los pacientes.

\section{REFERENCIAS}

1. Harapan $\mathrm{H}$, Itoh $\mathrm{N}$, Yufika A, Winardi W, Keam S, Te H, et al. Coronavirus disease 2019 (COVID-19): A literature review. J Infect Public Health. 2020;13(5):667-673. Available in: https://doi. org/10.1016/j.jph.2020.03.019

2. WHO coronavirus disease (COVID-19) dashboard. [cited 2020 December 10]. Available in: https://covid19.who.int/

3. Comunicado Técnico Diario COVID-19 México. [Fecha de acceso: 10 de diciembre de 2020]. Disponible en: www.gob.mx/cms/ uploads/attachment/file/598305/Comunicado_Tecnico_Diario_ COVID-19_2020.12.09.pdf

4. Pérez-Padilla R, Fernández R, García-Sancho C, Franco-Marina F, Mondragón E, Volkow P. Demand for care and nosocomial infection rate during the first influenza AH1N1 2009 virus outbreak at a referral hospital in Mexico City. Salud pública Méx. 2011;53(4):334-340. Disponible en: http://www.scielo.org.mx/scielo.php?script=sci_ arttext\&pid=S0036-36342011000400008\&lng=es

5. Organización Panamericana de la Salud (OPS). Teleconsulta durante una Pandemia [Fecha de acceso 03 de septiembre de 2020]. Accesible en: https://www.paho.org/ish/images/docs/covid-19-teleconsultas-es.pdf

6. World Health Organization. Telemedicine. Opportunities and developments in member states. Report on the second global survey on eHealth. Global Observatory for eHealth series. Volume 2. ISBN 9789241564144 ISSN 2220-5462@ World Health Organization; 2010. [cited 2012 July 08] Available from: http://www.who.int/goe/ publications/goe_telemedicine_2010.pdf

7. World Health Organization. Digital implementation investment guide: integrating digital interventions into health programmes. Geneva: World Health Organization; 2020. Available in: https://www.who.int/ publications/i/item/9789240010567

8. Hong Z, Li N, Li D, Li J, Li B, Xiong W, et al. Telemedicine during the COVID-19 pandemic: Experiences from Western China. J Med Internet Res. 2020;22(5):e1957. Available in: https://doi. org/10.2196/19577

9. Heyworth L, Kirsh S, Zulman D, Ferguson JM, Kizer KW. Expanding access through virtual care: the VA' s early experience with Covid-19. NEJM Catal Innov Care Deliv. 2020;1(4):1-11. Available in: https:// catalyst.nejm.org/doi/full/10.1056/cat.20.0327

10. Hollander JE, Carr BG. Virtually perfect? Telemedicine for COVID-19. N Engl J Med. 2020;382(18):1679-1681. Available in: https://doi. org/10.1056/nejmp2003539

11. Ohannessian R, Duong TA, Odone A. Global telemedicine implementation and integration within health systems to fight the COVID-19 pandemic: a call to action. JMIR Public Health Surveill. 2020;6(2):e18810. Available in: https://doi.org/10.2196/18810
12. Peine A, Paffenholz P, Martin L, Dohmen S, Marx G, Loosen SH. Telemedicine in Germany during the COVID-19 pandemic: multiprofessional national survey. J Med Internet Res. 2020;22(8):e19745. Available in: https://doi.org/10.2196/19745

13. Contreras CM, Metzger GA, Beane JD, Dedhia PH, Ejaz A, Pawlik TM. Telemedicine: patient-provider clinical engagement during the COVID-19 pandemic and beyond. J Gastrointest Surg. 2020;24(7):1692-1697. Available in: https://doi.org/10.1007/s11605020-04623-5

14. Centro Nacional de Excelencia Tecnológica en Salud. Acciones de Telesalud en Secretarías de Salud Estatales en México durante 2020. Ciudad de México: Secretaría de Salud; 2020. Accesible en: https://cenetec-difusion.com/observatoriotelesalud/telemedicinaen-mexico/

15. Méndez J, Llanos A. Presupuesto para salud mental, octubre 2020; CIEP, México, CIEP Investigaciones. [Diciembre de 2020] Recuperado desde: https://ciep.mx/presupuesto-para-salud-mental-relevanciaante-la-COVID-19/

16. Roldán-Gómez FJ, Jordán-Ríos A, Álvarez-Sangabriel A, GuízarSánchez C, Pérez-De Isla L, Lasses-Ojeda LA, et al. Telemedicina como instrumento de consulta cardiológica durante la pandemia COVID-19. Cardiovasc Metab Sci. 2020;31(Suppl: 3):259-264. doi:10.35366/93957.

17. Gobierno de la Ciudad de México. SEDESA COVID-19. [Diciembre de 2020] Recuperado desde: https://covid19.cdmx.gob.mx/

18. Smith AC, Thomas E, Snoswell CL, Haydon H, Mehrotra A, Clemensen $J$, et al. Telehealth for global emergencies: Implications for coronavirus disease 2019 (COVID-19). J Telemed Telecare. 2020;26(5):309-313. Available in: https://doi.org/10.1177/1357633×20916567

19. Hjelm NM. Benefits and drawbacks of telemedicine. J Telemed Telecare. 2005;11(2):60-70. Available in: https://doi. org/10.1258/1357633053499886

20. Saigí RF, Torrent SJ, Soler RI, Almazán SC, Kotzeva A, Villalobos HJ. Framework for the implementation of a telemedicine service. Washington, DC: PAHO; 2016. 2016. Available in: https://iris. paho.org/bitstream/handle/10665.2/28414/9789275119037_eng. pdf? sequence $=6$ \&isAllowed $=y$

21. Telehealth Quality Group EEIG - International Code of Practice for Telehealth Services 2018/19 v2). 2018-2019. Available in: https:// www.physioaustria.at/system/files/general/2018-19-internationaltelehealth-code-of-practice.pdf

22. Botrugno C. Towards an ethics fortelehealth. Nurs Ethics. 2019;26(2):357367. Available in: https://doi.org/10.1177/0969733017705004

23. Fleming DA, Edison KE, Pak H. Telehealth ethics. Telemed J E Health. 2009;15(8):797-803. Available in: https://doi.org/10.1089/ tmj.2009.0035

24. Miespera A, Ahonen SM, Reponen J. Ethical aspects of eHealthsystematic review of open access articles. FinJeHeW [Internet]. 2013;5(4):165-171. Available in: https://journal.fi/finjehew/article/ view/9401

25. World Medical Association Statement on the Ethics of Telemedicine. [Cited 2021 January 27] Available in: https://www.wma.net/policiespost/wma-statement-on-the-ethics-of-telemedicine/

Conflicto de intereses: Los autores declaran no tener conflicto de intereses. 